\title{
LA PROPIEDAD URBANA DE MONFORTE DEL CID A MEDIADOS DEL SIGLO XIX
}

\author{
M. J. Santos Deltell
}

\begin{abstract}
RESUMEN
La evolución de la urbanización de Monforte del Cid durante el siglo XIX presenta algunas características destacables:

-localización de los edificios mejor dotados en el centro urbano, denominado centro histórico. Siendo ésta la zona elegida para vivienda de las clases pudientes.

- una segunda parte del municipio estaría configurada por el ensanche, donde se encuentran las principales vías de acceso a la localidad.

- la periferia, por último, sería el lugar donde se ubican las clases trabajadoras.
\end{abstract}

\section{RÉSUMÉ}

L'évolution de l'urbanisation de Monforte del Cid pendant le XIX ${ }^{\text {ème }}$ siècle offrit des caractéristiques importants:

-localisation des principaux bâtiment du village au centre historique. C'est le lieu ou habitent les classes puissantes.

-la deuxième zone était configurée par l'agrandissement oú se trouvent les artéres d'accés á localitées prochaines: Agost, Elche, Alicante, etc.

-le troisième c'est la périphérie oú s'accomdent les travailleurs ruraux, des usines, etc.

En esta comunicación se aborda el estudio de la propiedad urbana en un municipio de la provincia, a mediados del siglo pasado, para determinar los usos y funciones del suelo en aquella época.

Durante el siglo XIX vamos a constatar el proceso de transición de la estructura urbana de Monforte, desde unas formas heredadas del pasado a otras que configurarán el paisaje de la ciudad moderna. La demanda de viviendas e infraestructuras van a remodelar el casco 
antiguo hasta congestionarlo en un principio, ensanchando el perímetro edificado, posteriormente, con la apertura de nuevas calles y con la creación de otros espacios.

Las fuentes de donde proceden los datos que hemos estudiado se encuentran en el Libro Padrón de Habitantes de 1870, del Archivo Municipal de Monforte (AMM) y del Libro Catastro de Riqueza Rústica y Urbana de 1853, también del mismo Archivo Municipal.

En el primero de ellos se encuentra una relación exhaustiva de todos los habitantes del pueblo, indicando el lugar de residencia, calles, número de policía, agrupados por familias, identificando el grado de parentesco, la edad, sexo, el lugar de nacimiento y la profesión. En algunos casos, hace también mención expresa de si son necesitados o mendicantes.

En el segundo, aparecen todas las calles del pueblo, con el número de casas de cada una, el valor del líquido imponible, lo que nos ha permitido señalar las distintas zonas socioeconómicas del núcleo urbano, así como también los sectores mejor dotados del municipio.

La población del casco urbano en 1860 era de 3.678 habitantes, el número de edificios era de 343, que se articulaban en torno a 20 calles, de disposición alargada unas, las que seguían los ejes del ensanche y otras, radioconcéntricas, alrededor de la Iglesia.

El valor medio por casa era de 83,95 reales, por encima de los 90 la mayoría se encontraban en la zona comprendida entre las calles Rabalet (sic), Parador, Carril, Huerta, Plaza de Santa Ana y Mayor, estando considerada aquélla como la de mayor renta inmobiliaria, suelo urbano con mayor poder especulativo y lugar de residencia de las clases acomodadas y profesiones liberales.

La tipología de las casas era semejante a la de casi todos los pueblos valencianos: casas de planta baja, adaptada a vivienda y "cambra" o cámara alta, destinada a guardar la cosecha del secano. La diferenciación social se acusaría más en el interior que en el exterior, aunque ésta era también visible si la casa constaba de una o varias plantas.

La mayoría del vecindario poseía una casa, con lo cual no existía gran concentración de la riqueza urbana en manos de unos pocos, sino que ésta se encontraba muy repartida; de todas formas, siempre había algunos considerados como los más ricos del pueblo, que poseían varias fincas en el núcleo.

Según el Padrón, la pequeña propiedad agraria era dominante en Monforte a fines del pasado siglo. El grupo de mayores contribuyentes urbanos lo constituían ricos propietarios con influencia social, que habían acumulado las propiedades que tenían como fruto de sucesivas herencias de padres a hijos.

Las funciones del espacio urbano se van a estudiar y situar en cada área municipal, ya que los datos que aparecen en el Catastro ofrecen la posibilidad, al consignar los propietarios de las casas de cada calle, sus referencias familiares, las profesiones de los mismos, y el origen y procedencia de las personas, con lo que también se puede esbozar un estudio de los movimientos migratorios, que sería de escasa importancia, dada la antigüedad de la fecha, pues es de todos conocidos que los trasvases de gentes suele ser un fenómeno social del siglo XX. La mayor parte del vecindario, por lo tanto, era natural del pueblo, aunque algunos procedían de migraciones interprovinciales.

Para una mejor comprensión del trabajo, lo acompañamos de un plano municipal, en el que dividimos la zona objeto de estudio en tres partes:

a) Núcleo antiguo: serían las calles que configuraban el primitivo emplazamiento del 
Cuadro 1

CALLES DE MONFORTE DEL CID $/ 853$

$n^{8}$ casas $\quad n^{0}$ prop. valor tot. valor med.
(líq. impon.)

a) Núcl. antig.

\begin{tabular}{lrrrc} 
ALTA & 17 & 23 & 870 & 51,1 \\
CARNICERIA & 14 & 15 & 1.191 & 85 \\
CRUZ & 12 & 21 & 840 & 70 \\
HUERTA & 25 & 30 & 2.658 & 106 \\
MAYOR & 31 & 393 & 271 & 105 \\
MORERIA & 18 & 26 & 1.035 & 57,5 \\
RABALET & 6 & 91 & 175 & 195,8 \\
SANGRE DE XTO & 16 & 22 & 1.095 & 68,4 \\
SANTA ANA & 6 & 9 & 900 & 150 \\
SANTA ELENA & 17 & 23 & 1.106 & 65 \\
SAN PASCUAL & 3 & 5 & 290 & 96 \\
\hline Totales: & 165 & 222 & 14.431 & $1.049,8 / 95,4$ \\
\hline
\end{tabular}

b) Ejes. ensan.

$\begin{array}{llllc}\text { AGOST } & 13 & 20 & 825 & 63,4 \\ \text { CARRIL } & 49 & 58 & 4.495 & 91 \\ \text { ELCHE } & 35 & 47 & 2.078 & 59,3 \\ \text { PARADOR } & 27 & 31 & 3.322 & 123 \\ \text { SALITRE } & 23 & 27 & 2.104 & 91\end{array}$

\begin{tabular}{lllll}
\hline Totales & 147 & 183 & 12.824 & $427,7 / 85,5$ \\
\hline
\end{tabular}

c) Periferia

\begin{tabular}{lrrll} 
CALVARIO & 10 & 15 & 540 & 54 \\
CUEVAS BAJAS & 6 & 8 & 360 & 60 \\
NORIAS & 3 & 5 & 135 & 45 \\
SAN ROQUE & 12 & 19 & 510 & 42.5 \\
\hline Totales & $3 \mathrm{~L}$ & 47 & 1.545 & $201.5 / 50,3$ \\
\hline
\end{tabular}

$\mathrm{N}^{0}$ total casas: 343

$\mathrm{N}^{\mathrm{N}}$ total propiet.: 452

Valor total: $28.800 \mathrm{rs}$.

Valor medio/casa: 83,95 rs.

(*) En la calle Cuevas Altas no habia ninguna casa; se trata de híbitat troglodítico. 
pueblo. Presentan trazados concéntricos, en torno a la fortaleza de la Iglesia, serían las calles: Cruz, Morería, Alta, Nueva, Sangre de Cristo, Huerta, Santa Elena, Mayor, Rabalet, Postigos, Plaza de Santa Ana, Plaza de San Pascual y Plaza Mayor.

b) Ejes del ensanche del municipio: se trata de los antiguos caminos hacia las poblaciones vecinas que a partir de ahora van a quedar integrados en el núcleo urbano, son calles largas donde la mayoría de las casas son nuevas. Estaría formada por las calles: Carril, Salitre, Agost, Elche y Plaza del Parador.

c) Anillo periférico: en general lo integran las calles situadas en el extrarradio. A este grupo pertenecen las calles de: Norias, Fábricas, San Roque, Calvario, Cuevas Altas y Cuevas Bajas.

En 1853 existían en el pueblo un total de 343 casas, repartidas entre 20 calles. El valor medio por casa era de 83,95 reales. Esta cifra estadística ofrece umbrales distintos entre unas zonas y otras. Así, por ejemplo, donde las casas alcanzaban mayores valores era en el núcleo antiguo: 95,4 rs. por término medio: seguidas por las de los ejes de ensanche, con 85,5 rs. por casa.

Sin embargo, y dentro del mismo núcleo urbano antiguo, las diferencias son notorias entre las calles situadas a la derecha de la Iglesia Parroquial (Cruz, Morería, Sangre de Cristo, Alta y Santa Elena), y las de la izquierda (Mayor, Huerta, Plaza de Santa Elena y Rabalet). El primer grupo de calles albergaba casas más humildes, donde ninguna de ellas sobrepasaba el tipo establecido de los 95,4 reales. El segundo, presentaba viviendas que en todos los casos superaban los 100 reales, destacando la calle Rabalet, donde había seis casas solamente cuyo valor medio se estimaba en casi 200 reales $(195,8)$.

En los ejes de ensanche, las mejores casas se sitúan en la plaza del Parador, con un valor medio de 123 rs. cada una. Le seguían a continuación, las que se ubicaban en la carretera Madrid-Alicante, cuyo eje longitudinal lo formaban las calles Salitre y Carril respectivamente, con valores próximos a los 100 rs. en ambos casos (91 rs.).

Por último, en la periferia municipal, el valor de las casas se reducía sensiblemente, siendo la media de 50,3 rs.

Cuadro II

MAYORES CONTRIBUYENTES DE MONFORTE 1853

\begin{tabular}{lll}
\hline CALLE & \multicolumn{1}{c}{ NOMBRE } & CONTRIBUCION \\
\hline & & \\
a) Núcl. antig. & Juan Beltrán & 5.113 rs. \\
HUERTA & Bartolomé Beltrán & 4.426 \\
HUERTA & Migual Juan Pujalte & 4.366 \\
HUERTA & Hrdos. M Martínez & 4.610 \\
MAYOR & Antonio Miralles & 5.630 \\
RABALET & & \\
b) Ejes Ensan. & & \\
CARRIL & Pascual Candela & 5.094 \\
CARRIL & Juan Soriano & 4.920 \\
PARADOR & Isidro Pujalte & 4.893 \\
\hline
\end{tabular}


Entre los mayores contribuyentes del municipio, con cantidades superiores a 4.000 rs., destaca el vecino Antonio Miralles, con 5.630 rs., que vivía en la calle Rabalet (núcleo antiguo). Le seguían Juan Beltrán, con 5.113 rs., que vivía en la calle Huertas, y Pascual Candela, con 5.094 rs., en la de Carril, próxima al Parador.

El resto de contribuyentes mayores, sobrepasaban todos ellos la cantidad de 4.000 rs. y se localizaban sus viviendas en la mejor zona el pueblo, esto es, en el exterior del núcleo antiguo y zona de contacto con los ejes del ensanche: concretamente, la calle Rabalet o Nueva, que debe su nombre a ser en otro tiempo el arrabal del núcleo urbano.

Según la distribución social de la riqueza, entre las tres partes en que hemos dividido el pueblo, sería la b), ejes del ensanche, la que contaría con un número absoluto mayor de propietarios censados $(102,73,18 \%)$.

\begin{tabular}{|c|c|c|c|}
\hline \multicolumn{4}{|c|}{$\begin{array}{c}\text { Cuadro III } \\
\text { DISTRIBUCIÓN SOCIAL }\end{array}$} \\
\hline CALLE & PROPIET. & JORNALEROS & MENDICANTES \\
\hline \multicolumn{4}{|l|}{ a) Núcleo antig. } \\
\hline NUEVA & 12 & 5 & \\
\hline MAYOR & 4 & 2 & \\
\hline POSTIGOS & 7 & 2 & \\
\hline Totales & $23(16,54 \%)$ & $9(4,5 \%)$ & \\
\hline \multicolumn{4}{|l|}{ b) Ejes ensanche } \\
\hline AGOST & 9 & 23 & \\
\hline CARRIL & 39 & 12 & \\
\hline ELCHE & 25 & 17 & \\
\hline PARADOR & 13 & I & \\
\hline SALITRE & 16 & 28 & \\
\hline Totales: & $102(73,18 \%)$ & $81(40,5 \%)$ & \\
\hline \multicolumn{4}{|l|}{ c) Periferia } \\
\hline CALVARIO & 8 & 12 & \\
\hline CUEVAS ALTAS & 29 & 7 & \\
\hline CUEVAS BAJAS & 1 & 28 & \\
\hline NORIAS & 1 & 24 & \\
\hline SAN ROQUE & 4 & 18 & \\
\hline Totales: & $14(10 \%)$ & $111(55,5 \%)$ & $7(100 \%)$ \\
\hline
\end{tabular}


Cuadro IV

OCUPACIONES Y PROFESIONES

\begin{tabular}{|c|c|c|}
\hline PROFES. LIBERALES & NÚM. & LOCALIZJCALLE. \\
\hline ABOGADO & 1 & Parador \\
\hline NOTARIO & 1 & Parador \\
\hline \multicolumn{3}{|l|}{ Comerciantes: } \\
\hline REVENDEDOR & 3 & Cuevas Altas y Bajas \\
\hline TENDERO & 11 & Parador, Cuevas, Elche \\
\hline VENDEDOR & 2 & Nueva y Carril \\
\hline \multicolumn{3}{|l|}{ Oficios } \\
\hline ALBAÑIL & 2 & Calvario y Carril \\
\hline ALBEITAR & 2 & Parador y Carril \\
\hline APERADOR & 4 & Parador, Salitre y S. Roque \\
\hline BARBERO & 1 & Parador \\
\hline CARDADOR & 1 & Agost \\
\hline CARPINTERO & 1 & Matyor \\
\hline CARRETERO & 11 & Agost, Carril, Rabalet, \\
\hline CORREONERO & 1 & Salitre \\
\hline COSEDOR & 9 & S. Roque, Salitre y Elche \\
\hline ESPARGATERO & 1 & Salitre \\
\hline ESQUILADOR & 1 & Cuevas Bajas \\
\hline ESTANQUERO & 1 & Parador \\
\hline ESTERERO & 2 & Agost y Salitre \\
\hline GUARDA & 1 & Rabalet \\
\hline GUARNICIONERO & 2 & Salitre y Agost \\
\hline HARINERO & 2 & Parador y Rabalet \\
\hline HERRERO & 4 & Carril, Parador y Salitre \\
\hline HILANDERO & 1 & Cuevas Altas \\
\hline HORNERO & 1 & Cuevas Bajas \\
\hline ORDINARIO & 2 & Parador y S. Roque \\
\hline PANADERO & 4 & Parador, S. Roque y Carril \\
\hline PASTOR & 6 & Norias, S. Roque y Agost \\
\hline PEON CAMINERO & 2 & Elche y Carril \\
\hline POSADERO & 1 & Parador \\
\hline PLEITISTA & 5 & Postigos, Cuevas Altas \\
\hline SERENO & 2 & Cuevas Altas \\
\hline TORNERO & 2 & Agost y Carril \\
\hline TEJEDOR & 2 & Elche, Parador \\
\hline TRATANTE & 5 & Mayor, Rabalet y Carril \\
\hline YESERO & 2 & Carril y Cuevas Bajas \\
\hline ZAPATERO & 1 & Cuevas Bajas \\
\hline Totales & 100 & \\
\hline
\end{tabular}


El grupo de jornaleros sería mayoritario en la tercera parte, c) o periferia, con un total de 111 hombres $(55,5 \%)$.

Los mendicantes vivirían estrictamente en esta tercera zona, generalmente en las cuevas. Representantes de profesiones liberales había en el pueblo dos, un abogado y un notario, ambos eran de Benidorm y residían en la plaza del Parador.

Los comerciantes eran $16(13,7 \%)$ del total de población activa. Se localizaban los comercios preferentemente en las salidas del pueblo, calles Carril, Elche y Parador; en las Cuevas vivían personas que ejercían el oficio de revendedores de objetos viejos y usados.

Como ocupación principal del municipio se registraba en aquellas fechas, el trabajo del esparto o pleita, con un número mayor de personas dedicadas a ello. Se contaba en este gremio con cinco pleitistas, dos estereros, un "espargatero" y nueve cosedores de esparto, lo que hacían un total de un $14,6 \%$ de mano de obra. Generalmente, los que se dedicaban a este menester eran de condición humilde y vivían en zonas poco favorecidas: Cuevas, Salitre y calle San Roque.

También contaba el pueblo con 11 carreteros $(9,4 \%)$, que elegían los accesos y salidas del núcleo urbano para el emplazamiento de su negocio.

Los demás trabajos estaban ampliamente representados con uno o dos individuos que se dedicaban al oficio, destacando con un número mayor: los pastores (6), tratantes (5), panaderos, herreros y aperadores (4).

Es de destacar el hecho de que algunos menesteres, como el de albeitar (veterinario), estanquero y ordinario, se localizaban en los puntos más céntricos del pueblo: calle Carril y plaza del Parador.

De las tres zonas en que hemos dividido el pueblo, la de mayor afluencia de inmigrantes corresponde a la b), ejes de ensanche, con 55,15\%, seguida de la zona c), periferia, con un $41,35 \%$, mientras que en el sector a) del núcleo antiguo, todos los habitantes son nacidos en Monforte, a excepción de uno que es de Novelda.

Respecto a la procedencia de estos inmigrantes, casi un $40 \%$ son oriundos de Novelda, debido a su proximidad; Elche y Monóvar arrojan saldos iguales, un 10\%; le siguen a continuación, Benidorm, Busot y Aspe, con un 6,8\%. Los demás municipios: Alicante, Agost, Castalla y San Vicente, en el ámbito provincial alicantino, con un 3,44\%; al igual que los procedentes de Tobarra (Albacete) y Albatera (Murcia), con un 3,44\%.

Como conclusiones, podemos apuntar las siguientes ideas:

- Las casas que alcanzaban valores mayores eran las de una zona del núcleo antiguo (izquierda de la Iglesia Parroquial), donde todas ellas superaban los 100 rs. de renta, destacando la calle Rabalet, donde había 6 casas solamente, cuyo valor medio se estimaba en casi 200 rs. -Los mayores contribuyentes vivían también en esta misma zona, como era el caso de Antonio Miralles, considerado el de mayor poder económico del pueblo, con una renta de 5.63o rs., que vivía en la calle Rabalet.

- Asimismo, en este sector predominaban los propietarios sobre los asalariados o jornaleros, con porcentajes de un 16,54\% para los primeros y un 4,5\% para los segundos.

-De manera general, y en relación a la procedencia de inmigrantes, en el núcleo antiguo, la mayor parte de los moradores eran naturales del municipio, no habiendo más que un inmigrante, del sexo femenino, que era de Novelda, que vivía en la calle Rabalet, por su unión matrimonial con un propietario local. 
Cuadro V

PROCEDENCIA DE INMIGRANTES

a) Núcleo antiguo

CALLE NUEVA (RABALET)

(Novelda) $\quad 3,44 \%$

b) Ejes ensanche

AGOST

4 (Monóvar,

Novelda,

Elche,

$S$ Vicente)

$13.79 \%$

CARRIL

5 (Alicante.

Elche 3,

Novelda)

$17,24 \%$

ELCHE.

2

(Elche,

Novelda)

$6.89 \%$

PARADOR

3

(Aspe 2,

Benidorm)

SALITRE

2

(Aspe.

Novelda)

$10,34 \%$

$6.89 \%$

c) Periferia

CALVARIO

(Novelda)

$6,89 \%$

CUEVAS ALTAS

(Castalla.

Monovar,

Tobarra,

Albatera)

(Novelda)

(Novelda 2,

NORIAS.

Busot.

Agost)

SAN ROQUE

(Monóvar)

$13,79 \%$

$3,44 \%$

- Respecto a la categoría socioprofesional de los habitantes, las profesiones liberales se localizaban preferentemente en la zona del Parador. 


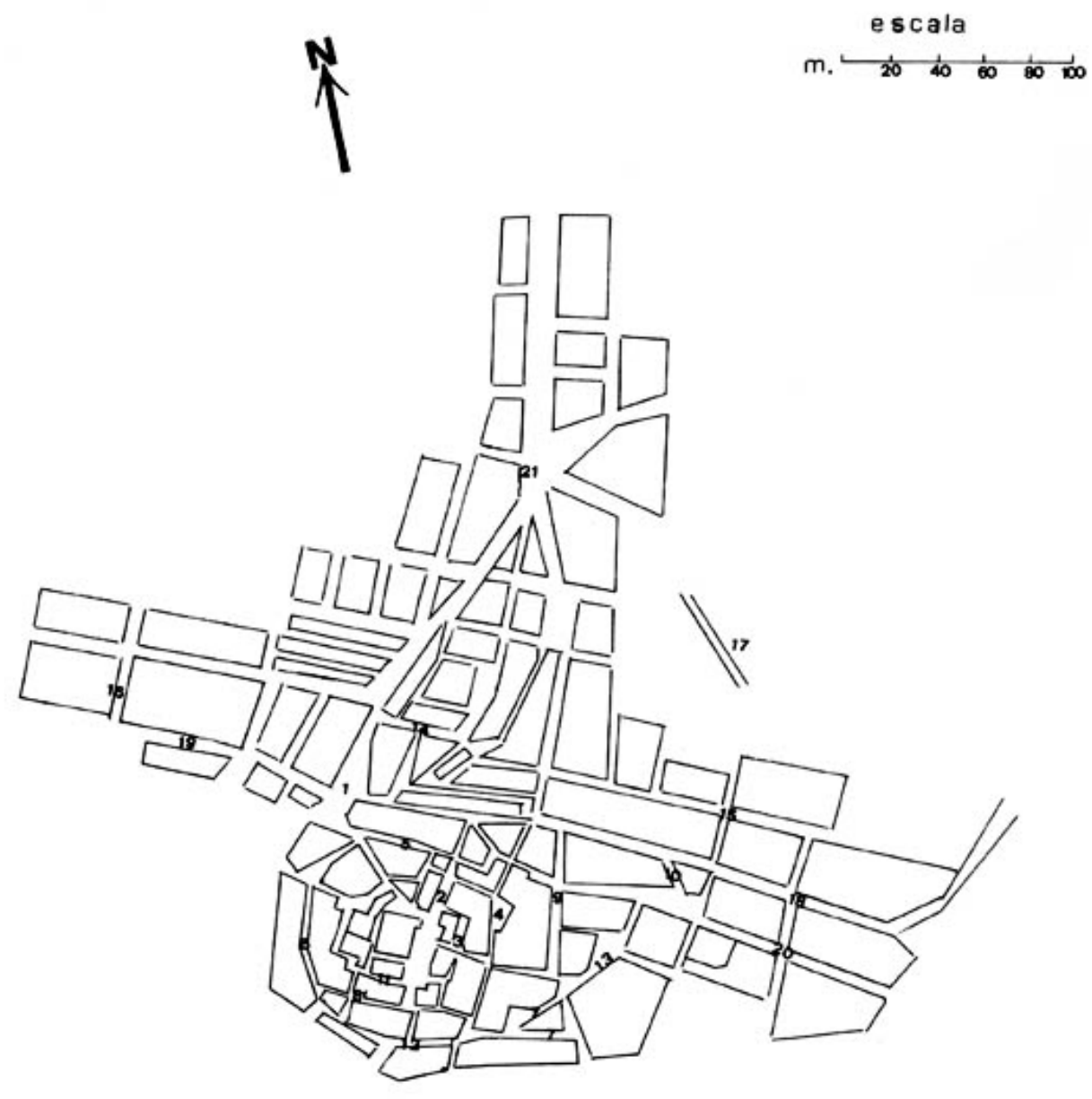

FIGURA 1: Plano de Monforte

Calles: 1, Parador. 2, Pza. Mayor. 3, Pza. de S. Pascual. 4, Pza. de Sta. Ana (Bonifacio Amorós). 5, C/ Mayor (Ramón y Cajal). 6, C/ de la Cruz. 7, C/ de la Sangre de Xto. 8, C/ de la Morería. 9, C/ Rabalet (Casto Richard). 10, C/ de los Postigos (J. Mirambell). 11, C/ Alta (Hernán Cortés). 12, C/ de Sta. Elena (Guillén Gras). 13, C/ de la Huerta (J. de la Torre). 14, C/ de S. Roque. 15, C/ del Calvario. 16, C/ de las Norias. 17, C/ de las Cuevas (Altas y Bajas). 18, C/ del Carril (Avda. de Alicante). 19, C/ del Salitre (Jorge Juan). 20, C/ de Elche (I. Pastor Casas). 21, C/ de Agost. 